Luis Felipe San Martín-Baeza'

José Alex Leiva-Caro²

1. Enfermero. Magíster en Salud Pública. Departamento de Salud Municipal. San Carlos. Chile.

2. Doctor en Ciencias de Enfermería. Grupo de Investigación en Envejecimiento. Profesor Asistente del Departamento de Enfermería. Profesor Magíster en Salud Pública. Facultad Ciencias de la Salud y de los Alimentos. Universidad del Bío-Bío. Chillán. Chile.

*Autor para correspondencia.

Correo electrónico: jleiva@ubiobio.cl (José Alex Leiva-Caro).

Recibido el 20 de agosto de 2018; aceptado el 5 de diciembre de 2018

\section{Relación entre salud cognitiva y adherencia farmacológica en adultos mayores con diabetes mellitus tipo 2}

\author{
Relation between Cognitive Health \\ and Pharmacological Adherence in \\ Older Adults with Type 2 Diabetes \\ Mellitus
}

\section{RESUMEN}

Objetivo: Evaluar la relación entre la salud cognitiva y la adherencia al tratamiento farmacológico en adultos mayores con diabetes mellitus tipo 2 de un centro comunitario de salud familiar de la provincia de Nuble, Chile. Método: Estudio analítico de corte transversal. La muestra estuvo constituida por 62 adultos mayores con diagnóstico de diabetes mellitus tipo 2. La recolección de datos se realizó mediante visitas domiciliarias utilizando los instrumentos Minimental

State Examination (MMSE), Test de Morisky Green Levine y un cuestionario de datos generales. Los datos fueron procesados en el programa SPSS, utilizando estadística descriptiva y analítica. El estudio contó con la aprobación de Comité de Bioética. Resultados: La adherencia al tratamiento farmacológico fue del 51,6\% ( $\mathrm{n}=32)$ y el $83,9 \%(n=52)$ se halló sin deterioro cognitivo. De los adultos mayores no adherentes al tratamiento farmacológico, el 46,7\% ( $\mathrm{n}=$ 14) presentó un nivel de hemoglobina glicosilada (HbA1c) del $7 \%$ o más. Se encontró relación entre la salud cognitiva y la adherencia al tratamiento farmacológico $(\mathrm{p}=0,004)$. Conclusiones: Se halló relación

entre la salud cognitiva y la adherencia al tratamiento farmacológico y una baja adherencia al tratamiento farmacológico en los adultos mayores con diabetes mellitus tipo 2. Cerca de la mitad de los adultos mayores no adherentes presentaron cifras de $\mathrm{HbAlc}$ superiores al 7\%.

PALABRAS CLAVE: Envejecimiento cognitivo, disfunción cognitiva, adhesión a la medicación, diabetes mellitus, adulto mayor.

\section{ABSTRACT}

Objective: To evaluate the relationship between cognitive health and adherence to pharmacological treatment in older adults with Type 2 Diabetes Mellitus from a Community Health Center of the Province of Nuble, Chile. Method: Cross-sectional analytical study, the sample consisted of 62 older adults with diagnosis of Type 2 Diabetes Mellitus. Data collection was performed through home visits using the Minimental State Examination (MMSE) instruments, Morisky Green Levine Test and a record of general data. The data were processed in the SPSS program, using descriptive and analytical statistics. The study was approved by the Bioethics committee. Results: Adherence to pharmacological treatment was $51.6 \%(\mathrm{n}=32)$, and $83.9 \%(\mathrm{n}=52)$ was found without cognitive impairment. Of the non-adherent older adults to the pharmacological treatment, $46.7 \%(n=14)$ had a level of glycosylated hemoglobin (HbA1c) of $7 \%$ or more. A relationship was found between cognitive health and adherence to pharmacological treatment $(\mathrm{p}=0.004)$. Conclusions: There was a relationship between cognitive health and adherence to pharmacological treatment and a low adherence to pharmacological treatment in older adults with Type 2 Diabetes Mellitus. Nearly half of non-adherent older adults had levels of glycosylated hemoglobin (HbA1c) above the normal range.

KEYWORDS: Cognitive aging, cognitive dysfunction, medication adherence, diabetes mellitus, aged.

\section{- INTRODUCCIÓN}

El deterioro de las condiciones de salud física y mental, entre otras, determina la aparición de múltiples enfermedades crónicas no transmisibles $\left(\right.$ ECNT), enfermedades que son notorias en los adultos mayores $(\mathrm{AM})^{1}$. A saber, cuanto mayor sea la edad de una persona, mayor probabilidad tiene de presentar ECNT, y entre las más frecuentes están la hipertensión arterial, la diabetes mellitus y los trastornos del sistema musculoesquelético 2 . De hecho, una de las ECNT que más se ha acentuado en el último tiempo es la diabetes mellitus, que se ha convertido en un relevante problema de salud pública ${ }^{3}$. Es así como, a nivel global, la prevalencia de diabetes mellitus aumentó del 4,7\% al 8,5\% del año 1980 al $2014^{4}$. En Chile, por su parte, se reporta una prevalencia del $12,3 \%$, donde destaca un aumento en el grupo de 65 ańos o más con un 30,6\% ${ }^{5}$. Por esta razón, en Chile, el Ministerio de Salud lleva a cabo el Programa de Salud Cardiovascular (PSCV), como estrategia fundamental en la compensación y seguimiento de la diabetes mellitus y otras ECNT, con el objetivo de disminuir la morbimortalidad asociada a las enfermedades cardiovasculares. 
Cabe señalar que es una enfermedad con diferentes formas de presentación, siendo la más frecuente la diabetes mellitus tipo 2, que constituye alrededor del $80 \%$ al $90 \%$ de los casos ${ }^{6}$.

Al respecto, la Organización Panamericana de la Salud establece que para su tratamiento es indispensable mantener un adecuado plan alimentario, ejercicio físico y hábitos saludables y, de ser necesario, complementar con hipoglucemiantes orales e incluso con administración de insulina ${ }^{7}$. Ahora bien, cerca del $30 \%$ de los AM utilizan cinco o más medicamentos por día ${ }^{8}$, por lo que es necesaria una adecuada adherencia, pues mantenerla es uno de los elementos fundamentales para el control metabólico ${ }^{9}$. La adherencia al tratamiento farmacológico es definida como el grado en que el comportamiento de un individuo, en términos de tomar medicamentos, coincide con lo prescrito por el médico ${ }^{10}$. En ese sentido, en el contexto internacional se ha reportado que en personas con diabetes mellitus tipo 2, la adherencia al tratamiento farmacológico varía entre un $38,5 \%$ y un $93,1 \%{ }^{11}$. Asimismo, en los AM se reporta que el $49,3 \%$ presenta una insuficiente adherencia al tratamiento farmacológico $^{12}$. Por su parte, en Chile se reporta que el 50,9\% de AM con diabetes mellitus tipo 2 cumple con la adherencia al tratamiento farmacológico ${ }^{13}$. Ahora bien, en Chile, la diabetes mellitus tipo 2 está incorporada al listado de Garantías Explícitas en Salud (GES), la cual garantiza acceso, oportunidad y protección financiera para todo paciente, independientemente de su previsión social, asegurando a través del Programa de Salud Cardiovascular el manejo oportuno por un equipo de salud multidisciplinario integrado principalmente por médico, nutricionista y enfermera/o. Por tanto, el seguimiento de los pacientes con diabetes se realiza a través de controles periódicos de 20 minutos cada 3 a 6 meses, dependiendo del riesgo cardiovascular. En los controles se busca detectar elementos de descompensación y dańo de órganos blancos, se verifican metas terapéuticas, se realiza prescripción de medicamentos, aplicación de pauta de prevención de úlceras del pie diabético, educación de insulinoterapia, refuerzo de adherencia al tratamiento farmacológico, evaluación nutricional y tratamiento dietoterapéutico. Adicionalmente, se les solicita una batería de exámenes de laboratorio cada 3 a 6 meses, electrocardiograma y examen de fondo de ojo en forma anual, y se brindan consultas podológicas cada mes, consejerías individuales y talleres grupales a aquellos pacientes con descompensación de su enfermedad.

Por lo anterior, esta temática se ha convertido en un tema prioritario de salud pública, sustentado, además, en el aumento de las tasas de hospitalización, los costos sanitarios y los fracasos terapéuticos ${ }^{14}$. En consecuencia, los AM diabéticos, al no mantener la adherencia farmacológica, pueden presentar hiperglicemias mantenidas, que con el tiempo causan complicaciones micro y macrovasculares, deterioro de la calidad de vida, dificultad para evaluar la efectividad de los medicamentos y elevados costos económicos ${ }^{15}$. Al respecto, se ha reportado que los costos totales asociados a la diabetes mellitus oscilan entre 141,6 millones de dólares y 174 mil millones de dólares ${ }^{16}$.

Ahora bien, con la edad, una de las barreras más importantes en los AM para lograr un adecuado nivel de adherencia al tratamiento farmacológico es el deterioro cognitivo, pues la adherencia requiere de un conjunto de procesos cognitivos ${ }^{17}$. En efecto, con la edad, diversas funciones cognitivas se comienzan a alterar, comenzando por el deterioro de la velocidad de procesamiento de la información, la memoria episódica, los procesos de control ejecutivo y la atención selectiva ${ }^{18}$. A saber, se ha reportado que la diabetes mellitus tipo 2 estaría asociada a una disminución de la función cognitiva, especialmente a nivel de la memoria y flexibilidad mental ${ }^{19}$. De igual forma, se ha encontrado asociada a una escasa cognición, especialmente en las funciones ejecutivas, aprendizaje y cognición global ${ }^{20}$, así como también la existencia de relación entre la hemoglobina glicosilada (HbA1c) y la pérdida de la función cognitiva ${ }^{21}$. En consecuencia, se propone realizar un estudio que busca evaluar la relación entre la salud cognitiva y la adherencia al tratamiento farmacológico en AM con diabetes mellitus tipo 2.

\section{- MATERIAL Y MÉTODO}

El estudio fue analítico de corte transversal; la población estuvo conformada por 90 AM con diabetes mellitus tipo 2 inscritos en el PSCV de un centro comunitario de salud familiar de la provincia de Nuble, Chile. La muestra correspondió al total de la población y el muestreo fue no probabilístico.

En cuanto a los criterios de elegibilidad, se incluyeron AM de 65 años o más con diabetes mellitus tipo 2 inscritos en el PSCV que tenían al menos un control el último año con médico, nutricionista y enfermero/a; estaban en tratamiento con hipoglicemiantes orales, y eran residentes de la zona urbana. Fueron excluidos: quienes estaban en tratamiento con insulina, hospitalizados, no sabían leer ni escribir, presentaban problemas de audición, visión o expresivos severos (voz inaudible, no articulada) y con diagnóstico de alguna enfermedad mental (objetivado con el carnet de control de salud). Para algunos de los criterios de inclusión y exclusión se utilizó la ficha clínica electrónica. Finalmente, se incluyeron $\mathrm{n}=62$ usuarios, lo que correspondió a la muestra, los mismos que representaron el 68,8\% de la población total. El 31,2\% $(\mathrm{n}=28)$ restante no pudo ser abordado por diferentes motivos: dirección incorrecta $(\mathrm{n}=$ 13), casa sin moradores después de 2 visitas $(n=6)$, fallecidos $(n=7)$ y hospitalizados $(\mathrm{n}=2)$.

Para la recolección de los datos se dispuso de un cuestionario de datos generales para abordar características sociodemográficas y de salud, y de dos instrumentos, que fueron: el Minimental State Examination versión modificada ${ }^{22}$ para evaluar la salud cognitiva y el Test de Morisky Green Levine $^{23}$, para evaluar la adherencia al tratamiento farmacológico.

Para obtener la información, se solicitó autorización formal en el centro comunitario de salud familiar. Una vez obtenida, se procedió a extraer datos de interés de los AM corroborando criterios de elegibilidad. Entre los datos se obtuvo información para contactarlos y el valor de la última HbA1c. El trabajo de campo fue llevado a cabo por el investigador principal en el mes de enero de 2018. Cuando fue posible, se coordinó la visita por medio de una llamada telefónica con el fin de disminuir las visitas fallidas, corroborando además criterios de elegibilidad. El día de la visita se invitó a participar al AM, previa explicación del objetivo del estudio; la aceptación a participar quedó manifiesta por medio del consentimiento informado. Los datos fueron recolectados en la siguiente secuencia: solicitud del carnet de control del PSCV, para verificar antecedentes generales y presencia de algún criterio de exclusión; luego se aplicó el cuestionario de datos generales, seguido del Minimental State Examination y el Test de Morisky Green Levine. Una vez finalizada la recogida de datos se entregaron recomendaciones generales sobre adherencia al tratamiento farmacológico.

En cuanto a las consideraciones éticas, el estudio contó con la aprobación del Comité de Bioética y Bioseguridad de la Universidad del Bío-Bío.

El análisis de los datos se realizó en el paquete estadístico Statistical Package for The Social Sciences (SPSS) versión 15.0 para Windows, y se utilizó estadística descriptiva y analítica. Para lo descriptivo se utilizaron frecuencias absolutas, porcentajes, media aritmética y desviación típica. Para las correlaciones se realizaron pruebas de normalidad de Kolmogórov-Smirnov; luego se estimó la relación entre la salud cognitiva y la adherencia al tratamiento farmacológico con prueba de $\chi^{2}$, cuando 
el valor esperado en alguna celda fue cero o menor que 5 en la prueba exacta de Fisher. Se consideró un nivel de significación $\leq 0,05$.

\section{- RESULTADOS}

En cuanto a las características sociodemográficas de los AM, la media de edad fue de 75,44 (DE 6,0) años; respecto al sexo, el 64,5\% ( $\mathrm{n}=$ 40) correspondió a mujeres; por su parte, el $48,4 \%(\mathrm{n}=30)$ vivía con al menos una persona en su casa; y el $64,5 \%(n=40)$ tenía educación básica incompleta. En cuanto a las características de salud, el promedio de ańos diagnosticados con diabetes mellitus tipo 2 fue de 8,52 (DE 5,8 años; el consumo promedio de medicamentos por día fue de 5,16 (DE 2,5) y la media del nivel de HbA1c fue de 7,1 (DE 1,2). En cuanto a las variables principales del estudio, se observó que el $51,6 \%(n=32)$ presentó adherencia al tratamiento farmacológico, y el 83,9\% ( $\mathrm{n}=52)$ se encontró sin deterioro cognitivo. Ahora bien, el 46,7\% $(n=14)$ de los AM no adherentes al tratamiento farmacológico tenían en su mayoría entre 75 y 84 años, el 73,3\% ( $n=22)$ fueron mujeres, el $43,3 \%(n=13)$ vivía con al menos una persona en su hogar y el 56,7\% $(\mathrm{n}=17)$ tenía un nivel de educación básica incompleta. Además, el $80 \%(\mathrm{n}=24)$ de los AM no adherentes al tratamiento farmacológico tenían 3 años o más de diagnóstico de diabetes mellitus tipo 2, el $50 \%(\mathrm{n}=15)$ tomaban cinco o más medicamentos al día; el 46,7\% ( $n=14)$ tenía tres o más ECNT y el $46,7 \%(\mathrm{n}=14)$, un nivel de HbA1c del $7 \%$ o más. En cuanto al objetivo del estudio, se halló relación entre la adherencia al tratamiento farmacológico y la salud cognitiva $\left(\chi^{2}\right.$ de Pearson $\left.=8,267, p=0,004\right)$.

\section{- DISCUSIÓN}

El estudio responde al objetivo, al encontrar relación entre salud cognitiva y adherencia al tratamiento farmacológico en AM con diabetes mellitus tipo 2 de un centro comunitario de salud familiar, y aporta información descriptiva sobre las variables de interés en esta población.

Respecto a las características sociodemográficas, se halló que la media de edad es similar a lo reportado en estudios a nivel nacional ${ }^{24} \mathrm{e}$ internacional $^{25}$, lo que pone de manifiesto el impacto que esta enfermedad tiene en un grupo etario económicamente inactivo, con las implicaciones que ello significa en el sistema sanitario, social y financiero. En cuanto al sexo, las mujeres predominaron en el estudio, lo cual concuerda con un reporte a nivel nacional ${ }^{5}$, donde se indica que la prevalencia de diabetes mellitus es mayor en dicho grupo. También es similar a lo descrito en otros estudios ${ }^{3,26}$, existiendo consenso sobre esta situación, que podría deberse a que las mujeres asisten regularmente a los centros de salud, por lo que pueden ser pesquisadas con mayor facilidad respecto a los hombres y, por tanto, sugiere la necesidad de seguir creando nuevas estrategias para ampliar la detección de la enfermedad en hombres, los cuales pueden tener esta patología. En cuanto al número de personas con las que vivían los $\mathrm{AM}$, se encontró que en mayor porcentaje viven con una persona, lo que coincide con lo descrito en la literatura especializada ${ }^{19}$, reafirmándose que en esta etapa del ciclo vital comúnmente se produce la marcha de los hijos o la muerte del cónyuge, lo que puede generar en las personas un reducido apoyo en la administración de medicamentos, pues se sabe que un grupo social cumple una función esencial en el desarrollo de la persona, la sobrevivencia y estabilidad de la sociedad. Respecto al nivel educacional, lo hallado en el estudio permitió evidenciar una gran disparidad. Esto es debido a que la mayoría de los AM tenían un nivel educacional de básica incompleta, lo cual es similar a otro reporte ${ }^{24}$, lo que sustenta la idea de que el bajo nivel educacional que tienen los AM en cierta medida refleja las mayores brechas de acceso y oportunidad de educación que existían en décadas anteriores, generando actualmente personas con menos ańos de estudios, lo que puede tener relación con la correcta comprensión de las indicaciones de tratamiento, entre ellas los medicamentos prescritos.

$\mathrm{Al}$ analizar la distribución de las características de salud, se observó que los AM tenían en promedio 8,5 años diagnosticados con diabetes mellitus tipo 2, lo que si bien se asemeja con un estudio ${ }^{27}$, es mayor que lo encontrado en otro ${ }^{20}$. Lo relevante es que se reafirma el carácter crónico de la enfermedad, con la consecuente necesidad de requerir medicamentos de forma permanente. Ahora bien, el número de medicamentos que consumían diariamente los AM fue, en promedio, de 5,2, cifra que es superior a lo reportado en otros estudios ${ }^{3,25}$; no obstante, en todos se destaca la polifarmacia, como una problemática en el cumplimiento correcto del tratamiento farmacológico. En efecto, cerca del $50 \%$ de los AM tenían tres ECNT, lo que es similar a otro estudio ${ }^{28}$ en donde se enfatiza que los AM diabéticos generalmente tienen otras enfermedades asociadas, lo que provoca un mayor número de medicamentos consumidos por día y dificulta aún más la correcta adherencia al tratamiento farmacológico. Ahora bien, se encontró además que los AM presentaron un aceptable control metabólico de la diabetes mellitus tipo 2, al hallar un nivel promedio de HbA1c del 7,1\%, situación positiva para el sistema sanitario, y similar a lo reportado en otro estudio realizado en personas con esta misma patología ${ }^{29}$. Este resultado refleja en parte el trabajo realizado por los profesionales de la salud, pues tienen entre sus objetivos que las personas con diabetes mantengan este parámetro lo más cercano al 7\%, dadas las complicaciones micro y macrovasculares en diversos órganos del cuerpo que se pueden producir cuando el nivel sobrepasa lo esperado. Sin embargo, llama la atención que a pesar de encontrarse una baja adherencia al tratamiento farmacológico, el nivel de compensación de la enfermedad según la HbAlc se puede considerar como aceptable.

Con respecto a la salud cognitiva, en su mayoría los AM del estudio no presentaron deterioro cognitivo, resultado que difiere de lo reportado a nivel nacional ${ }^{30}$, lo que en cierta medida se puede deber a la versión del instrumento utilizado, pues en este estudio se utilizó la versión modificada del MMSE, validada en la población chilena. A nivel internacional se reportó ${ }^{31}$ un resultado similar; no obstante, la literatura especializada no informa de una prevalencia única en cuanto a dicha condición, ya que la variación de esta se puede atribuir a los diferentes parámetros clínicos que puedan utilizar los investigadores, como también el rango de edad, el nivel de estudio, el tamaño muestral, los instrumentos utilizados o la sensibilidad de estos; por tanto, lo encontrado invita a los profesionales de la salud a realizar mejoras en las estrategias utilizadas al momento de entregar indicaciones y educación respecto a la terapia farmacológica en los AM.

Por otra parte, se observó que los AM presentaron una prevalencia de adherencia al tratamiento farmacológico disminuida. Esto es similar a otros reportes en población chilena ${ }^{13,24}$, y gradualmente diferente de estudios realizados a nivel internacional ${ }^{32,33}$; no obstante, el porcentaje se enmarca en lo que describe la literatura ${ }^{11}$. Lo importante es la alarmante cifra de pacientes con baja adherencia al tratamiento farmacológico, situación que podría repercutir en el control metabólico, en los costos directos de medicamentos, intervenciones sanitarias y en general muchos otros inconvenientes en la salud pública de la sociedad.

$\mathrm{Al}$ analizar la adherencia al tratamiento farmacológico según la edad, el mayor porcentaje de AM no adherentes se encontró en el rango de 75 a 84 años, así como también fue más prevalente en mujeres, situación que concuerda con lo reportado en un estudio a nivel nacional ${ }^{24}$, lo que refleja que la disminución de diversas funciones mentales, físicas y socia- 
les producto de la edad puede incapacitar la realización de actividades de la vida diaria como tomar los medicamentos; mientras que sobre la baja adherencia farmacológica en las mujeres, la revisión realizada no reportó una respuesta clara sobre esta situación. Sin embargo, podría atribuirse a que como la prevalencia de la diabetes mellitus tipo 2 es mayor en ellas, en consecuencia, son mayormente entrevistadas sobre esta condición respecto de los hombres. En cuanto a la adherencia al tratamiento farmacológico según el número de personas con las que viven los AM, lo encontrado fue similar a lo reportado por otro estudio ${ }^{28}$, en relación a que aquellos que viven con una persona en su hogar tuvieron niveles más bajos de adherencia al tratamiento farmacológico, lo cual se puede deber a la disminución de figuras de apoyo que tienen los AM en la autogestión de sus cuidados, determinando insuficientes ideas o estrategias para tomar de forma correcta sus medicamentos. A su vez, la adherencia al tratamiento farmacológico también fue más baja en los AM con un nivel de educación básica incompleta, similar a otro estudio ${ }^{3}$. En consecuencia, la educación puede ser un factor protector en la autogestión de los cuidados de salud; adicionalmente, se encontró que los AM presentaron un porcentaje menor de adherencia al tratamiento farmacológico en aquellos que tenían tres o más años diagnosticados con diabetes mellitus tipo 2, tres ECNT asociadas y tomaban cinco o más medicamentos diariamente, resultados que son similares a los de otros estudios ${ }^{3,34}$, que concuerdan en que con los ańos tienden a aparecer patologías que requieren un mayor número de medicamentos, evidenciado por la polifarmacia, lo mismo que ha sido reportado en este estudio y que podría contribuir a la inadecuada adherencia al tratamiento farmacológico. Respecto a la adherencia al tratamiento farmacológico según el nivel de $\mathrm{HbAlc}$, se apreció una menor adherencia al tratamiento farmacológico cuando tenían un nivel de HbA1c $\geq 7$, lo que difiere de otro estudio ${ }^{28}$, donde se encontró que la HbAlc no se relaciona con la falta de adherencia. Sin embargo, lo observado es interesante, puesto que los AM que no se tomaban los medicamentos empeoraban su control metabólico, por lo que sería atractivo investigar estas características con estudios más específicos y con un mayor número de personas en la muestra. Por otro lado, se evidencia la importancia de mantener la correcta adherencia al tratamiento farmacológico, dada la menor descompensación metabólica que se produciría en estos pacientes.

Ahora bien, en el estudio se encontró relación entre la salud cognitiva y la adherencia al tratamiento farmacológico. Esto, por un lado, difiere del estudio realizado por Villacís ${ }^{28}$, lo que se puede deber a algunas características de la población estudiada, como por ejemplo área geográfica de residencia, uso de insulinoterapia, ayuda en la toma de medicamentos, complicaciones y comorbilidades, así como también a la versión del ins- trumento utilizado y sus características psicométricas. Por otro lado, la relación encontrada se ve respaldada por lo reportado por $\mathrm{Cuevas}^{30}$, quien manifiesta que es indispensable considerar el estado de salud cognitiva de los AM en el momento de prescribir medicamentos y educar sobre ellos. En ese sentido, la relevancia de la relación observada es un dato útil para los profesionales de la salud, pues los orienta sobre cómo generar nuevas estrategias que permitan mejorar la adherencia al tratamiento farmacológico considerando la salud cognitiva como un componente central de las intervenciones educativas. La información también es útil para reorientar los programas de salud, poniendo énfasis en la importancia de la evaluación cognitiva en todos los AM diabéticos que requieran de tratamiento farmacológico, incluyendo a la familia como parte importante en su terapia. Sin duda que estos datos también son relevantes, al brindar un área de oportunidad en investigación sobre la adherencia al tratamiento farmacológico y permiten aportar información nueva en población chilena sobre la salud cognitiva en los AM y adherencia farmacológica.

En cuanto a las limitaciones del estudio, se consideran el tamaño de la muestra y la selección de los participantes, que correspondió a un muestreo no probabilístico, por lo que los datos no pueden ser generalizables. Entre las recomendaciones se sugiere continuar con estudios sobre las variables abordadas, siendo longitudinales y con diferentes diseños, incorporando otras variables de interés como pueden ser el apoyo familiar, la polifarmacia, las comorbilidades, considerando AM con uso de insulina.

\section{- CONCLUSIONES}

El estudio contribuye con datos que permiten comprender de mejor forma la adherencia al tratamiento farmacológico, al encontrar relación entre la salud cognitiva y la adherencia al tratamiento farmacológico. Además, se halló una baja adherencia al tratamiento farmacológico en los AM con diabetes mellitus tipo 2, lo que da cuenta de un preocupante problema de salud pública y refuerza la importancia de generar nuevas políticas públicas enfocadas en revertir dicha situación, debido a las múltiples consecuencias que esta puede provocar. Finalmente, destaca que cerca de la mitad de los AM no adherentes presentaron cifras de HbAlc por encima del 7\%, lo que manifiesta el efecto del no cumplimiento de la terapia farmacológica, al que se puede agregar el no cumplimiento de otras indicaciones que forman parte del tratamiento de la patología

\section{Conflicto de intereses}

Los autores de este trabajo declaran no tener ningún conflicto de intereses.

\section{- BIBLIOGRAFÍA}

1. Manton KG, Corder L, Stallard E. Chronic disability trends in elderly United States populations: 1982-1994. Proc Natl Acad Sci USA 1997:94(6):2593-98

2. Silguero S, Martínez M, Llanos A, Juncos G, Romero L, Abizanda P. Enfermedad crónica, mortalidad, discapacidad y pérdida de movilidad en ancianos españoles: estudio FRADEA. Rev Esp Geriatr Gerontol. 2014:49(2):51-8.

3. Ahmad NS, Ramli A, Islahudin F, Paraidathathu T. Medication adherence in patients with type 2 diabetes mellitus treated at primary health clinics in Malaysia. Patient Prefer Adherence 2013;17(7):525-30.

4. Organización Mundial de la Salud. Informe Mundial Sobre la Diabetes. Resumen de Orientación; 2016. [Internet]. [citado 07 de agosto de 2017]. Disponible en: http://apps.who.int/iris/bitstream/10665/204877/1/WHO_NMH_NVI_16.3_spa.pdf?ua=1

5. Ministerio de Salud, Gobierno de Chile. Encuesta Nacional de Salud 2016-2017 Primeros resultados. Departamento de Epidemiología,
División de Planificación Sanitaria, Subsecretaría de Salud Pública, Santiago 2017. [Internet]. [citado 16 agosto de 2017]. Disponible en: http://www.minsal.cl/wp-content/uploads/2017/11/ENS-201617 PRIMEROS-RESULTADOS.pdf

6. Goldstein BJ, Mueller-Wieland D. Type 2 Diabetes: Principles and Practice, 2. ${ }^{\text {a }}$ ed. Boca Raton, FL: Taylor \& Francis Group 2007.

7. Pan American Health Organization. Guías ALAD de diagnóstiCo, control y tratamiento de la Diabetes Mellitus Tipo 2. Estados Unidos, OPS 2009. [Internet]. [citado el 26 de agosto de 2017]. Disponible en: http://www.paho.org/hq/dmdocuments/2010/ Guias_ALAD_2009.pdf

8. Kim J, Parish AL. Polypharmacy and Medication Management in Older Adults. Nurs Clin North Am. 2017:52(3):457-68.

9. Martínez-Domínguez GI, Martínez-Sánchez LM, Lopera-Valle JS Vargas-Grisales, N. La importancia de la adherencia terapéutica. Rev Venez Endocrino Metab. 2016;14(2):107-16.
10. Conthe P, Márquez Contreras E, Aliaga Pérez A, Barragán Garcia B, Fernández de Cano Martin MN, González Jurado M, et al. Treatment compliance in chronic illness: Current situation and future perspectives. Rev Clin Esp. 2014;214(6):336-44.

11. Krass I, Schieback P, Dhippayom T. Adherence to diabetes medication: a systematic review. Diabet Med. 2015;32(6):725-37.

12. Núñez A, Montiel A, Martín E, Torres B, Lara C, González J. Adherencia al tratamiento en pacientes polimedicados mayores de 65 años con prescripción por principio activo. Aten Primaria. 2014;46(5):238-45.

13. Alcaíno D, Bastías N, Benavides C, Figueroa D, Luengo C. Cumplimiento del tratamiento farmacológico de la Diabetes Mellitus tipo 2 en adultos mayores: influencia de los factores familiares. Gerokomos. 2014;25(1):9-12.

14. Gutiérrez-Angulo ML, Lopetegi-Uranga P, Sánchez-Martín I, Garaigordobil-Landazabal M. Cumplimiento terapéutico en pacientes con hipertensión arterial y diabetes mellitus 2. Rev Calid Asis. 2012;27(2):72-7 
15. Ortiz M, Ortiz E, Gatica A, Gómez D. Factores psicosociales asociados a la adherencia al tratamiento de la Diabetes Mellitus Tipo 2 Ter Psicol. 2011:29(1):5-11.

16. Ng CS, Lee JY, Toh MP, Ko Y. Cost-of-illness studies of diabetes mellitus: a systematic review. Diabetes Res Clin Pract. 2014;105(2):151-63

17. Hudani ZK, Rojas CH. A scoping review on medication adherence in older patients with cognitive impairment or dementia. Res Socia Adm Pharm. 2016;12(6):815-29.

18. Ballesteros S. Envejecimiento Saludable: Aspectos Biológicos, Psi-

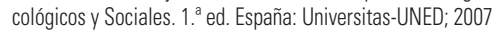

19. Miranda-Félix PE, Valles-Ortiz PM, Ortiz-Félix RE. Relación del control glucémico, función cognitiva y las funciones ejecutivas en e adulto mayor con Diabetes Tipo 2: Revisión sistemática. Enferm Glob. 2016;15(42):472-80.

20. Salak Djokić B, Spitznagel MB, Pavlović D, Janković N, Parojčić A $\|$ lić $\mathrm{V}$, et al. Diabetes mellitus and cognitive functioning in a Serbian simple. J Clin Exp Neuropsychol. 2015;37(1):37-48.

21. Hernández M. Relación entre la función cognitiva y valores de hemoglobina glicosilada en pacientes diabéticos que acudían a la consulta de medicina interna de un consultorio de Guada Lacau 2012-2013. Valencia, 2013. [Internet]. Disponible en: http://mriuc bc.uc.edu.ve/bitstream/handle/123456789/1061/M.\%20Hernandez.pdf? sequence $=1$
22. Quiroga P, Albala C, Klaasen G. Validación de un test de tamizaje para el diagnóstico de demencia asociada a edad, en Chile. Rev Méd Chile. 2004:132(4):467-78

23. Val Jiménez A, Amorós Ballestero G, Martínez Visa P, Fernández Ferré ML, León Sanromá M. Estudio descriptivo del cumplimiento del tratamiento farmacológico antihipertensivo y validación del test Morisky y Green. Aten Primaria. 1992;10(5):767-70.

24. Bello N, Montoya P. Adherencia al tratamiento farmacológico en adultos mayores diabéticos tipo 2 y sus factores asociados. Gerokomos. 2017;28(2):73-77.

25. Leiva-Caro JA, Salazar-González BC, Gallegos-Cabriales EC, Gómez-Meza MV, Hunter KF. Relación entre competencia, usabilidad, entorno y riesgo de caídas en el adulto mayor. Rev. Latino-Am. Enfermagem. 2015;23(6):1139-48.

26. Sánchez-Cruz JF, Hipólito-Lóenzo A, Mugártegui-Sánchez SG, Yáñez-González RM. Estrés y depresión asociados a la no adherencia al tratamiento en pacientes con Diabetes Mellitus tipo 2. Aten Fam. 2016;23(2):43-7.

27. Nerat T, Locatelli I, Kos M. Type 2 diabetes: cost-effectiveness of medication adherence and lifestyle interventions. Patient Prefer Adherence. 2016;10:2039-49

28. Villacís G. Relación entre deterioro cognitivo y adherencia al tratamiento farmacológico en pacientes con Diabetes Mellitus tipo 2 que acuden al club de diabéticos del Hospital Humberto del Pozo de la ciudad de Guaranda en el año 2015. Ecuador; 2016. [Internet]. Disponible en: http://repositorio puce edu ec/handle/22000/10413

29. Feil DG, Zhu CW, Sultzer DL. The relationship between cognitive impairment and diabetes self-management in a population-based community sample of older adults with Type 2 diabetes. J Behav Med. 2012;35(2):190-9.

30. Cuevas H. Assessment of Cognitive Function in Type 2 Diabetes. J Nurse Pract. 2017;13(7):311-15.

31. Kinoshita T, Shimoda M, Sanada J, Fushimi Y, Hirata Y, Irie S, et al. Association of $\mathrm{GA} / \mathrm{HbA1c}$ ratio and cognitive impairment in subjects with type 2 diabetes mellitus. J Diabetes Complications. 2016;30(8):1452-55.

32. Li CL, Chiu YC, Bai YB, Lin JD. Stanaway F Chang HY. The co-occurrence of depressive symptoms and cognitive impairment and its relationship with self-care behaviors among community dwelling older adults with diabetes. Diabetes Res Clin Pract 2017:129:73-8.

33. Jaam M, Ibrahim M, Kheir N, Awaisu A. Factors associated with medication adherence among patients with diabetes in the Middle East and North Africa region: A systematic mixed studies review. Diabetes Res Clin Pract 2017:129:1-15.

34. Ramos Y, Morejón R, Gómez M, Reina M, Rangel C, Cabrera Y. Adherencia terapéutica en pacientes con diabetes mellitus tipo 2. Rev Finlay. 2017;7(2):89-98 